\title{
Semantic Models for the Geological Mapping Process
}

\author{
Vincenzo Lombardo ${ }^{1,2(凶)}$, Fabrizio Piana ${ }^{2,3}$, Dario Mimmo ${ }^{3,4}$, Enrico Mensa $^{1}$, \\ and Daniele P. Radicioni ${ }^{1}$ \\ 1 Dipartimento di Informatica, Università di Torino, Torino, Italy \\ vincenzo.lombardo@unito.it \\ 2 Consiglio Nazionale delle Ricerche, Istituto di Geoscienze e Georisorse, Torino, Italy \\ ${ }^{3}$ Gi-RES srl, Torino, Italy \\ 4 Dipartimento di Scienze della Terra, Università di Torino, Torino, Italy \\ http://www.di.unito.it/〜 vincenzo
}

\begin{abstract}
The geologic mapping process requires the organization of data according tothe general knowledge about the objects in the map, namely the geologic units, and tothe objectives of a graphic representation of such objects in a map, following some established model of geotectonic evolution. Semantics can greatly help such a process in providing a terminological base to name and classify the objects of the map and supporting the application of reasoning mechanisms for the derivation of novel properties and relations about the objects of the map.

The OntoGeonous initiative has built a terminological base of geological knowledge in a machine-readable format, following the Semantic Web tenets and the Linked Data paradigm, with the construction of an appropriate data base schema that can be then filled with the objects of the map. The paper will present the conceptual model of the geologic system and how the elements of the cartographic database are classified from general definitions. Also, the paper addresses the setup of web-based services that respond to queries concerning the properties of the map elements that are not explicitly asserted in the underlying data base, but are inferred through a reasoning process.
\end{abstract}

Keywords: Ontology · Geomapping · OntoGeonous · Geologic knowledge

\section{Introduction}

Modern geological maps, which are supported by large geo-databases and are implemented through interactive representations on WebGIS services, need explicit geological assumptions for their design and compilation. This task has been supported by the use of controlled vocabularies, such as those belonging to GeoSciML (GeoScience Markup Language), published by the IUGS CGI

(C) Springer International Publishing AG 2017

F. Esposito et al. (Eds.): AI*IA 2017, LNAI 10640, pp. 295-306, 2017.

https://doi.org/10.1007/978-3-319-70169-1_22 
Commission $^{1}$ and the INSPIRE "Data Specification on Geology" directives ${ }^{2}$. Recently, there has been an interest toward the semantic models, in order to provide a formal and interoperable representation of the geologic knowledge. The effective encoding of geologic knowledge through the formal languages of the semantic web technologies can improve the consistency of the represented knowledge and benefit from the inferential system that automatically produces novel knowledge [3]. The semantic approaches are fundamental for the integration and harmonization of geological information and services across cultural (e.g. different scientific disciplines) and/or physical barriers (e.g. administrative boundaries). A semantic approach to data representation (referring to existing ontologies and vocabularies) was used as an essential tool for providing data interoperability, as recently done at a transnational scale by $[6,13]$. Initiatives such as GeoSciML and INSPIRE, as well as the recent terminological shepherding of the Geoscience Terminology Working Group (GTWG), have been promoting information exchange of the geologic knowledge, providing the authoritative standard for geological knowledge encoding. The interconnection between the geologic knowledge in its various forms (such as geologic events, units, morphologies, etc.) and the several knowledge sources (such as lithological materials, geochronologic units, etc.) can be addressed through the recourse to the Linked Data paradigm $[4,10]$.

The OntoGeonous initiative has built a terminological base of geological knowledge in a machine-readable format, following the Semantic Web tenets and the Linked Data paradigm [14,15,21]. The major knowledge sources are GeoSciML schemata and vocabularies and INSPIRE directives. The Linked Data paradigm has been exploited by linking the already existing machinereadable encoding for some specific domains, such as the lithology domain and the geochronologic time scale. Finally, for the upper level knowledge, shared across several geologic domains, OntoGeonous resorted to NASA SWEET ontology. The OntoGeonous initiative has also produced a wiki that explains how the geologic knowledge has been encoded from shared geoscience vocabularies ${ }^{3}$. In particular, the sections dedicated to axiomatization support the construction of an appropriate data base schema that are filled with the elements of a map.

This paper describes how the semantic encoding of the geologic knowledge in an ontological format has contributed to the realization of the Piemonte Geological $\mathrm{Map}^{4}$ [21] and how semantic services have been built to answer prototypical questions posed by the geologists. In the next two sections, we review the related work on semantics for geological knowledge and sum up the OntoGeonous initiative. Then, we describe how the ontological knowledge has served the task

\footnotetext{
${ }^{1}$ Commission for the Management and Application of Geoscience Information (CGI) of the International Union of Geological Sciences (IUGS).

${ }^{2}$ An operative simplification of GeoSciML, published by INSPIRE Thematic Working Group Geology of the European Commission.

${ }^{3}$ https://www.di.unito.it/wikigeo/.

${ }^{4}$ http://arpapiemonte.maps.arcgis.com/apps/webappviewer/index.html?id=fff17326 6afa4f6fa206be53a77f6321.
} 
of constraining the map legend and implementing the classification criteria of the map elements. Finally, we present the web-based application schema and the first prototype designed to answer basic questions that require the reasoning services, overcoming the limitations of the underlying data base.

\section{Related Work}

Semantics-informed interpretation of datasets in the context of geomapping tasks has been addressed in three types of literature works: the technical infrastructures for semantics-informed applications, the ontological encoding of specialized domains, and the creation of controlled vocabularies (such as GeoScienceML and INSPIRE).

The technical infrastructures are very numerous in the geomatic literature. They are generally complementary approaches to OntoGeonous: where they introduce technical systems for realizing services, OntoGeonous introduces actual knowledge to support those services, also including consistency checking and automatic classification, which currently lack. Geon ${ }^{5}$ is a cyber-infrastructure for the integration of 3D- and 4D-data, where formal ontologies (SWEET, among others) are used to coordinate and integrate conceptual schemas of heterogeneous geological maps (cf. [18]). OpenEarth Framework and OpenTopography, both developed from Geon, are a semantics-based toolsuite for integration and visualization of multi-dimensional data [17], and a high-resolution topographic data application ${ }^{6}$. AuScope ${ }^{7}$ is an integrated Australian national framework [26] that allows real time access to data, information and knowledge, stored in distributed repositories. For querying geological maps, AuScope uses vocabularybased services which overcome differences in geoscience terms due to language, synonyms and local variations. SETI (Semantics Enabled Thematic data Integration) [7] is a system that enables the retrieval of information from thematic data archives via semantic search (including the development of ontologies for the classification schemata and the integration of several applications). Finally, Ma's ontology mentioned above is the subject of the pilot interactive multimedia project developed by [19], which provided an animated visualization of this ontology and interaction functions over the ontology, the animation and an online geologic map, in synchronization with the RDF-based Geologic Time Scale ontology.

Approaches aimed at the ontological encoding of specialized domains are Virtual Solar-Terrestrial Observatory (VSTO) and Space Physics Archive Search and Extract (SPASE). $\mathrm{VSTO}^{8}$ is a semantic data framework based on an ontology of the domains of solar physics, space physics and solar-terrestrial physics [8].

\footnotetext{
${ }^{5}$ http://www.geongrid.org/.

${ }^{6}$ http://www.opentopography.org/.

7 https://www.researchgate.net/publication/234183449_AuScope's_use_of_Standards_ to_Deliver_Earth_Resource_Data.

${ }^{8}$ https://www.vsto.org.
} 
As in the case of OntoGeonous, VSTO also refers to the functional decomposition of SWEET, reusing, e.g., the notions of earth and sun realms, respectively. The SPASE consortium ${ }^{9}$ is an international group of space physics researchers, Virtual Observatory developers and data providers who have created a comprehensive space physics data model, converted into an OWL ontology, consisting of terminology, definitions, and protocols for the documentation of a data product [20]. These approaches employ ontological encoding of specialized domains; as such, ontologies are a commodity for terminology and documentation rather than an effective machine-interpretable knowledge.

Finally, there are a number of approaches that make the effort of relying on authoritative resources (such as GeoScienceML), without introducing ad hoc knowledge specifications. All these approaches currently make a very basic use of ontological encoding: OntoGeonous improves such methods by providing a comprehensive approach to the formal encoding of the geologic knowledge, aimed at subsequent automatization of application algorithms. Examples of these approaches are OneGeology ${ }^{10}$, an international initiative of the geological surveys in the world. Its goal is to create a worldwide geological map by harmonizing data from different providers, using GeoSciML standard, TaxonConcept ${ }^{11}$ [12], which stores Open Nomenclature synonymy lists in the field of taxonomic classification of fossil species, The United States Geoscience Information Network ${ }^{12}$ a federated information-sharing framework that uses GeoSciML as data transfer standard [24].

The OntoGeonous approach, described in this paper, integrates the knowledge sources in a machine-readable format and applies the encoded knowledge to the geomapping task, also proposing a software architecture for its exploitation.

\section{Conceptual Modeling for Geologic Knowledge: The OntoGeonous Initiative}

The geologic mapping process, which produces the map, requires the organization of data according to the general knowledge about the objects in the map, namely the geologic units, and to the objectives of a graphic representation of such objects in a map, following some established model of geotectonic evolution. Semantics can greatly help such a process in two concerns: on the one hand, it provides a terminological base to name and classify the objects of the map; on the other, the machine-readable encoding of the geologic knowledge base supports the application of reasoning mechanisms and the derivation of novel properties and relations about the objects of the map [14,15].

OntoGeonous is an initiative of the University of Turin and the Institute of Geosciences and Georesources of the Italian National Research Council (CNRIGG) for the construction of a terminological knowledge base that is, on the

\footnotetext{
${ }^{9}$ http://www.spase-group.org/.

10 http://portal.onegeology.org/OnegeologyGlobal/.

11 http://taxonconcept.stratigraphy.net/.

12 http://usgin.org/.
} 
one hand, specific enough to classify the instances of a specific geodatabase, concerning the entries of the Geological Map of Piedmont and, on the other hand, general enough to connect all the knowledge required for the classification of the instances. OntoGeonous has produced a merged set of computational ontologies that has been realized through the OWL encoding of the statements reported in authoritative resources (see Fig. 1). In particular,

- GeoScience Markup Language (GeoSciML) ${ }^{13}$ a standard data interchange format supporting structures for geologic and earth science information, expressed in a number of UML schemata (classes, features, attributes, associations) and statements in natural language, for the major core geological concepts;

- INSPIRE (Infrastructure for Spatial Information in the European Community) ${ }^{14}$, a EU Commission directive of the Thematic Working Group Geology, aiming at creating a European Union spatial data infrastructure which will enable the sharing of environmental information among public sector organizations; INSPIRE encoding is based on a simplification of GeoSciML (GeoSciML+INSPIRE cookbook, addressing the major vocabularies) and is expressed through natural language statements;

- SWEET (Semantic Web for Earth and Environmental Terminology) ${ }^{15}$, developed by NASA-Jet Propulsion Laboratory since 2002, a set of ontologies in OWL format that represent a knowledge base for environmental and Earth system science terms [2,23], considered for major upper, generic concepts;

- vocabularies of specific subdomains of geologic knowledge that are relevant for the geomapping task, developed by the IUGS/CGI IWG Concept Definition Task Group ${ }^{16}$ : for example, we have imported the lithology domain vocabulary named Simple Lithology ${ }^{17}$, and the ICS Geological Time Scale Ontology [18] as a subtaxonomy of the Geochronologic Unit class of SWEET Representation. There were many cross-reference issues to address during the encoding: for example, the Geochronologic Unit class of OntoGeonous referes to SWEET GeologicTimeUnit class (actually the hierarchical path Representation - NumericalEntity - Interval - Duration - GeologicTimeUnit).

The core of the geologic knowledge is a taxonomy rooted by Geologic Feature, which encompasses all the geologic core knowledge, related to (1) MappedFeature, i.e. the spatial extent of the geologic feature on the map, (2) GeoChronologicUnit, root of the ICS GTS taxonomy, (3) CGIVocabularyTerm (an OntoGeonous taxonomy for CGI vocabularies), which provide specific concepts for the several subdomains, such as the ones for the earth materials, and to the

${ }^{13}$ Version 4.0 (2015), http://www.geosciml.org.

${ }^{14}$ D2.8.II.4 INSPIRE Data Specification on Geology - Technical Guidelines v. 3.0. (10.12.2013) ( http://inspire.jrc.ec.europa.eu/documents/Data_Specifications/ INSPIRE_DataSpecification_GE_v3.0.pdf).

15 https://sweet.jpl.nasa.gov/.

16 http://resource.geosciml.org/vocabulary/cgi/201211/.

17 http://resource.geosciml.org/vocabulary/cgi/201211/simplelithology.rdf. 
abstract descriptions in GeoSciML, which encode attributes, such as, the unit thickness. GeologicFeature is subdivided into four sub-taxonomies, namely GeomorphologicFeature (the landforms), GeologicUnit (the bodies of some material), GeologicStructure (configurations or patterns in which the geologic units are arranged), GeologicEvent (relevant events in geology).

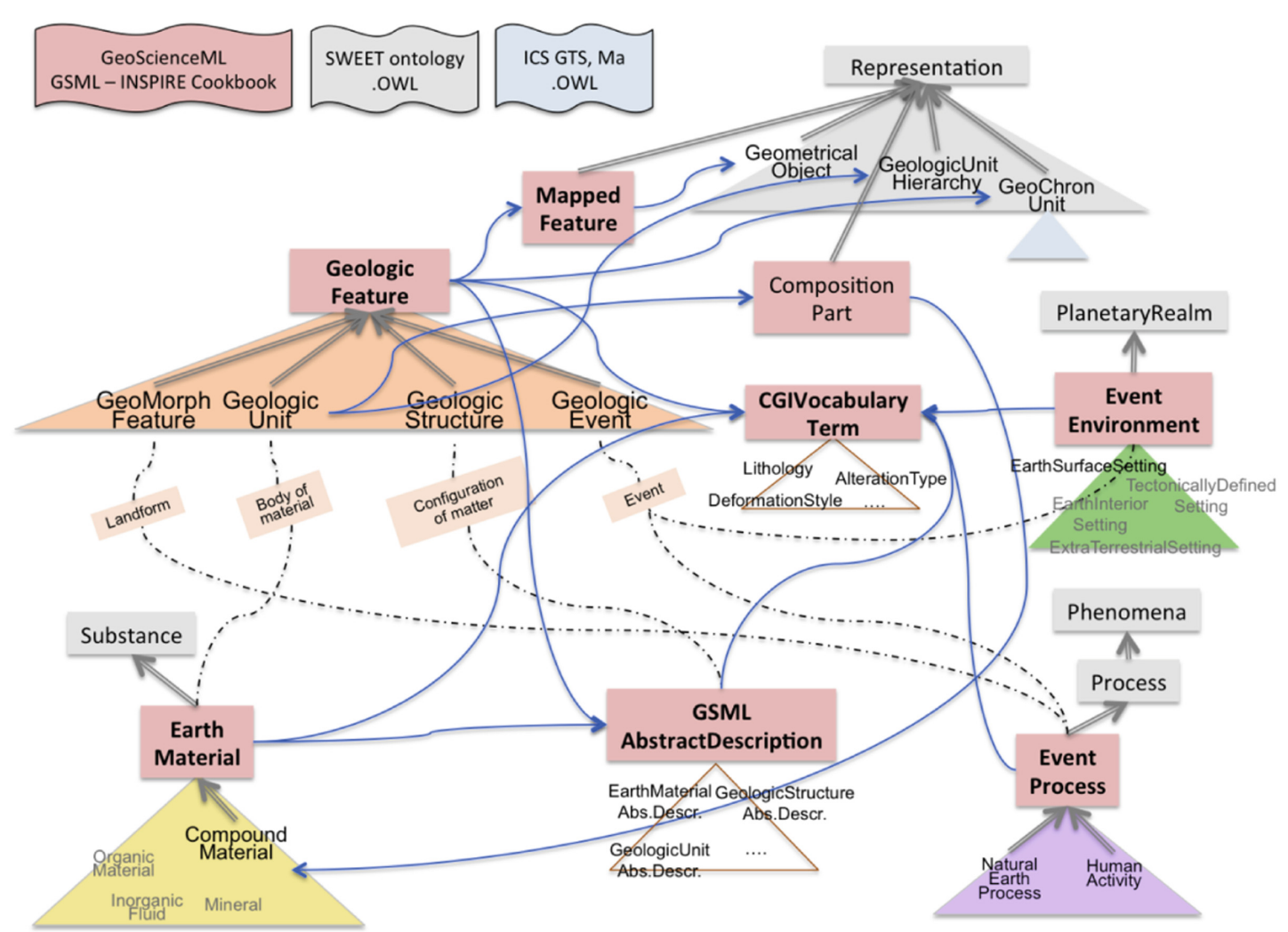

Fig. 1. The major taxonomies of OntoGeonous and their connections.

\section{Application of Semantic Knowledge to a Cartographic Project}

The Piemonte Geological Map [21] is grounded on a regional-scale Geodatabase consisting of some hundreds of Geologic Units that have several thousands instances in the Map [22]. Each Geologic Unit in the map is bounded by some Geologic Structures. The structure of the Geodatabase is grounded on the hierarchy of the Geologic Units, their characteristics (description) and their lithology (Simple Lithology encoded classes). The relations between the GeoDB contents (raws and columns describing the properties of the Mapped Features) and the general knowledge (concepts) stored in OntoGeonous are illustrated in Fig. 2 through a specific example, where links between the two knowledge sources are evidenced by arrowed lines. A specific geologic unit (Baldissero Formation) is 
a Lithostratigraphic unit made up of two parts (CP1 and CP2 represented by lithotypes) belonging to a higher rank geologic unit, the Synthem (i.e. the BTP3 Synthem) (as reported in the GeoDB). This Geologic Unit has also relations with a more general and conceptual knowledge framework, here represented by the Earth Material class (a Taxonomic Class of the GeoSciML Data Model) and the Simple Lithology controlled vocabulary (resources of the IUGS CGI Interoperability Working Group). These two knowledge sources are ideally linked with the GeoDB by arrowed lines, namely the Lithology and Geol_Unit3 columns of the GeoDB table.

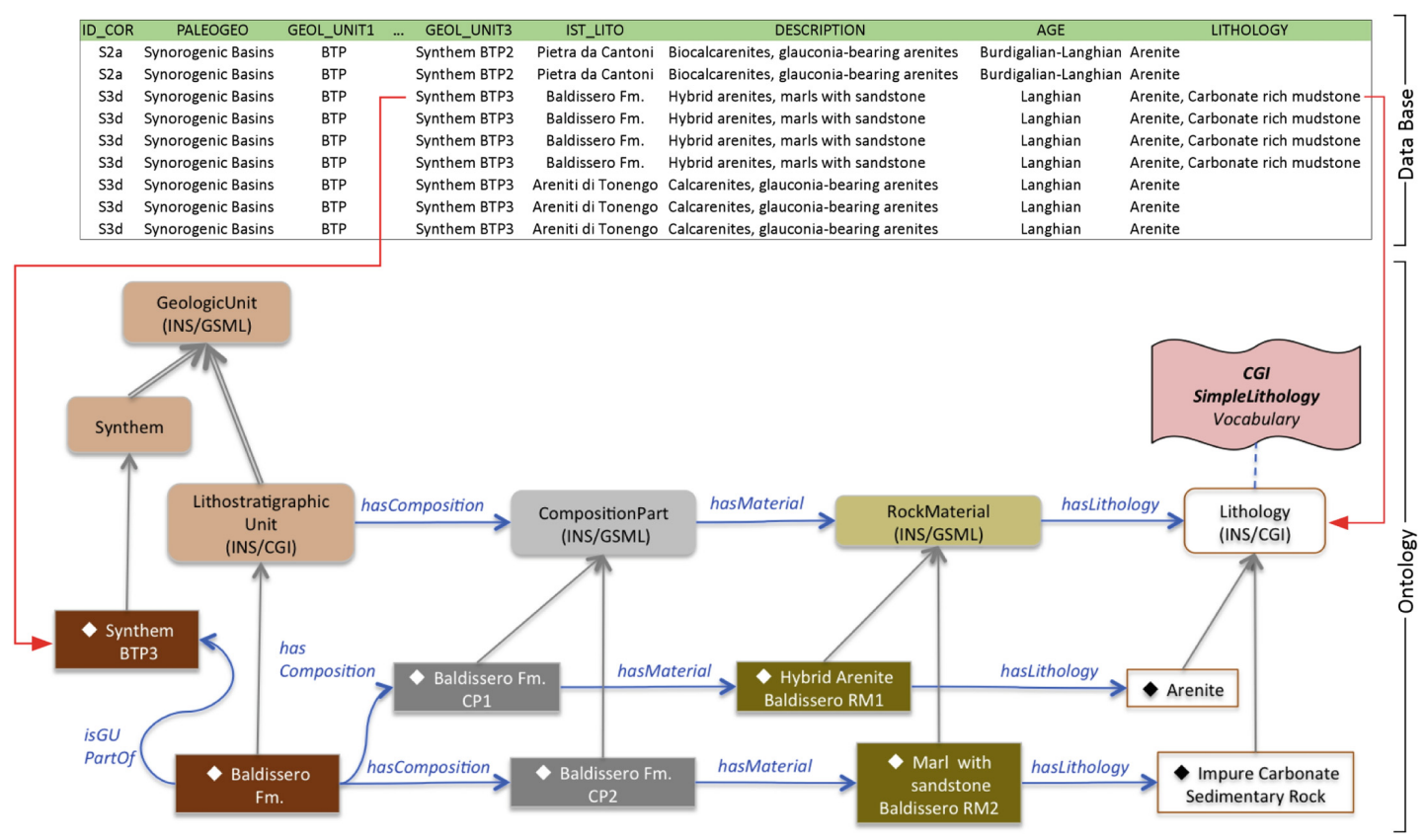

Fig. 2. A specific Geologic Unit (Baldissero Formation) is classified as a Lithostratigraphic unit made up of lower rank geologic units (compositional parts CP1 and CP2) represented by lithotypes belonging to a higher rank geologic unit, the BTP3 Synthem. This Geologic Unit has relations with a more general knowledge (represented in the Earth Material class, see Fig. 2) and is described by the CGI Simple Lithology vocabulary, as reported in the Geodatabase column Lithology.

In Fig. 3, we illustrate an application schema that can be deployed into several subtasks: the knowledge base OntoGeonous (an .owl file, possibly interconnected with other knowledge sources in the web, upper middle) resides in a server that hosts a service which is able to respond to queries posed about the encoded knowledge. The server runs on a physical machine equipped with four 2.30 GHz Intel(R) Xeon(R) CPUs and 65 GB RAM, executing a Scientific Linux 7.3 (Nitrogen) operating system. The described architecture is implemented as a 
Java program using the Jena framework; ${ }^{18}$ inference is performed based on the HermiT reasoner ${ }^{19}$, which is accessed through the OWL API interface ${ }^{20}$.

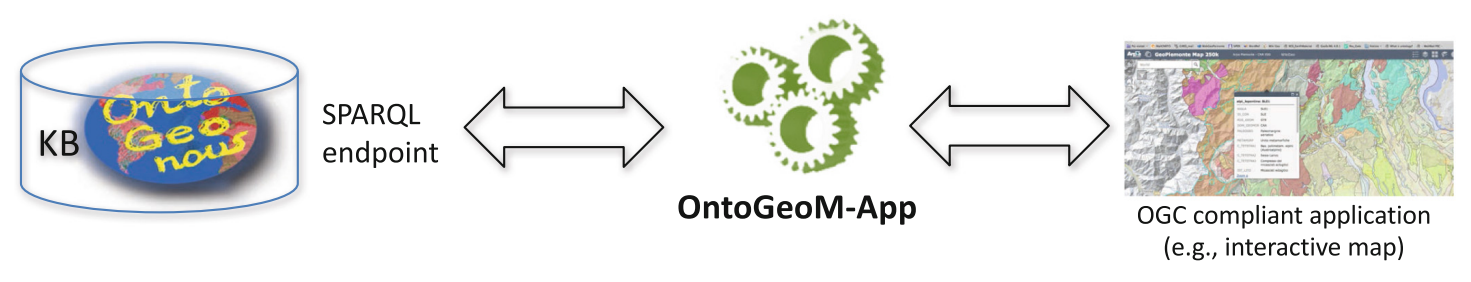

Fig. 3. The application schema for OntoGeonous server.

The specific application, generally named OntoGeoM-App in the figure, for implementing its specific task, poses queries to the knowledge base. We have currently implemented a prototype interface for querying the content of the instantiated knowledge base through a human-friendly query language, which works as an interface to semantic server that handles class expression queries. The query interface consists of a triple, addressing a subject (intended as "Find all entities XXX such that ..."), a property (intended as “... this property YYY valued as ..."), and an object (intended as "... ZZZ, the value of the property YYY"). Figure 4 reports the results for the query

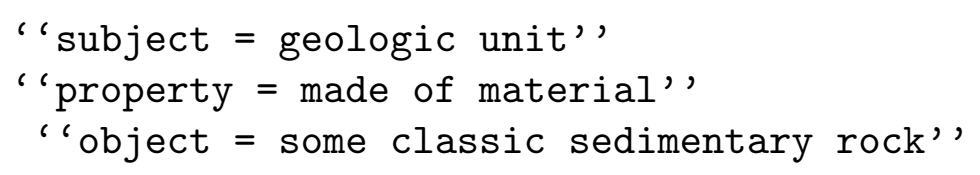

expressed in natural language as "Find any GEOLOGIC UNIT such that ... is composed of some part that consists of some lithological material that is ... some classic sedimentary rock." and translated into the class expression query

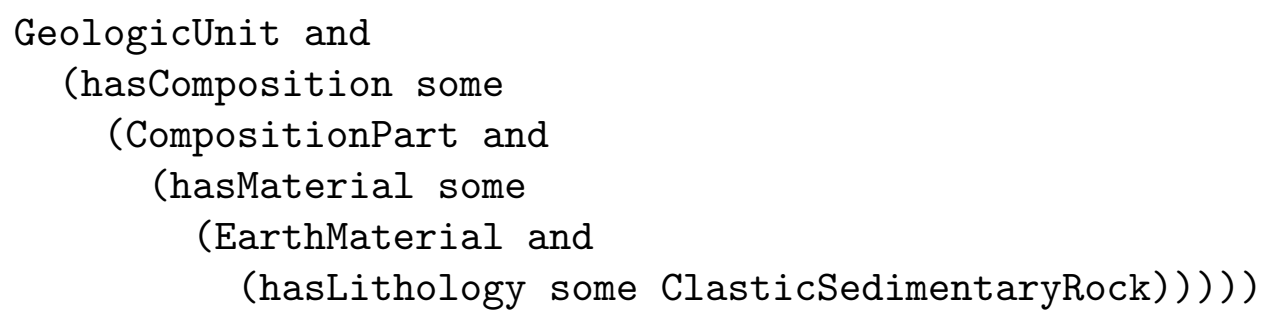

Several applications can be developed on this skeleton. For example, if we want to build a more detailed geological map (the current one is at $1: 250 \mathrm{~K}$ scale), we need an application that can fill automatically a number of entries in the data base by performing some effective reasoning on the knowledge base (after queries posed in the SPARQL language); or, for example, if we want to support

\footnotetext{
${ }^{18}$ Available at the URL https://jena.apache.org.

19 http://www.hermit-reasoner.com.

${ }^{20}$ http://owlapi.sourceforge.net.
} 
the cartographer with a draft map coloring based on some features of the units that are included in the knowledge base, such as the geochronologic reference and/or their lithology, the application can implement a map coloring algorithm informed by the semantic knowledge queried through the SPARQL endpoint.

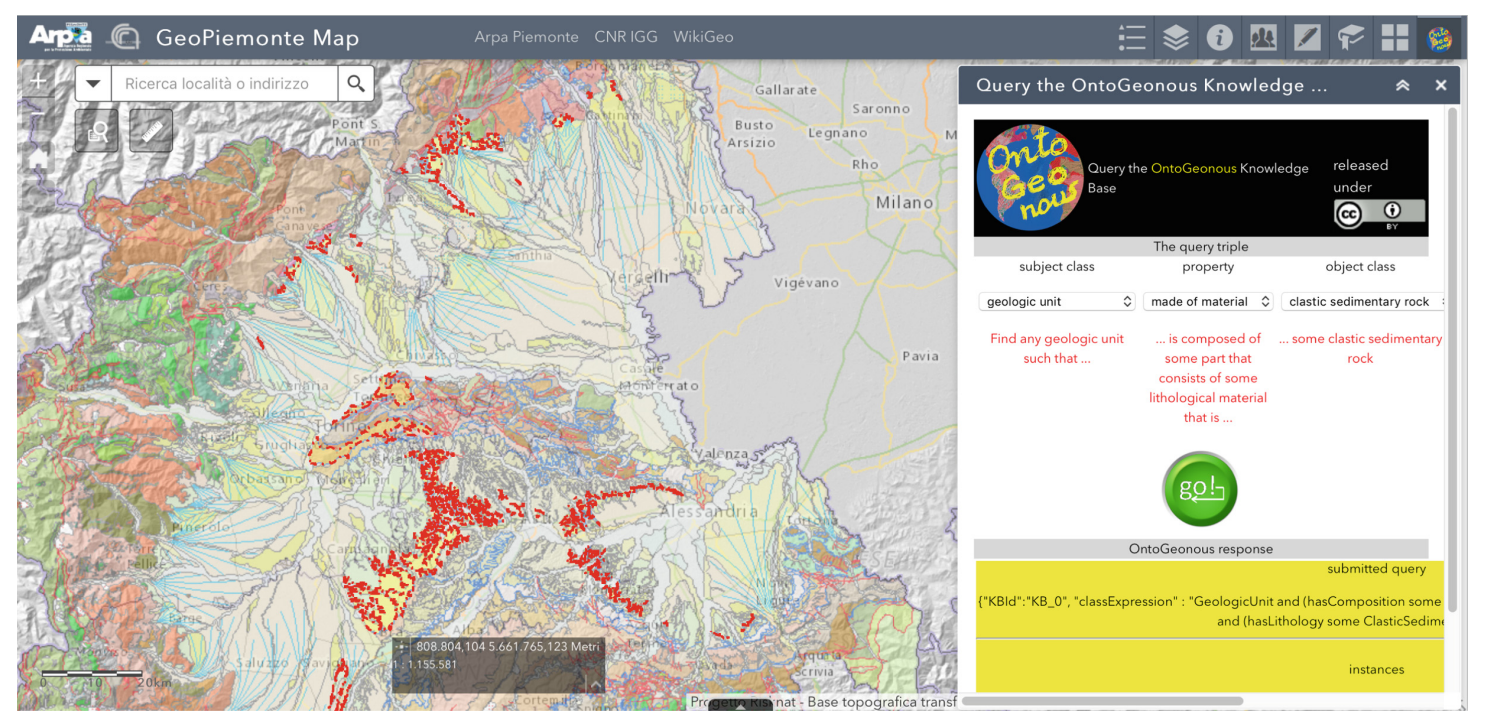

Fig. 4. The interface of the web app to query the map knowledge.

\section{Lessons Learned and Conclusions}

This paper has presented a machine-readable encoding of geologic knowledge, in the paradigm of the Semantic Web and the Linked Data, and its application to an actual geomapping task, namely the definition and the legend compilation of a cartographic map of the geologic units in a regional area. We have also seen how applications and web services can be developed from the encoded knowledge, accessible through a semantic server. In the rest of this section (and paper), we report on the lesson learned from the encoding of the geologic knowledge and its application of the geomapping process.

In general, the ontological encoding has supported the investigation on the epistemological nature of the relations existing between the different orders of Geological units. In particular, the ontological encoding has moved the terminological problem from the intuitive conception of relations to motivated and coherent sets of instances, assuring the re-traceability of the knowledge path (steps) followed during the implementation of the synthetic geological model, as required by the geomapping task. For example, the reasoner has classified the Lithostratigraphic class under the Lithologic class, so the ontology has induced a taxonomic relation upon the flat vocabulary of geologic unit terms.

The clear explicitation and formal encoding of the intended meaning of the concepts strongly constrain the definition and even the accuracy of the spatial location of the mapped features [25]. This improves the applicability of the 
knowledge "stored" in the geodatabase across several domains (e.g. geology, geophysics, geo-engineering, geo-environmental sciences, etc.), overcoming problems due to the use of different taxonomies. The analysis of the meaning of the geological knowledge chunks, as well as their disambiguation through the encoding into axioms, can cause the deprecation of some concepts. For instance, concept "Tectonostratigraphic Unit", largely used in the geological literature although with slightly different meanings, was deprecated. Indeed, concept "Tectonostratigraphic Unit" is a powerful tool that geologists often use to compare/distinguish portions of a sedimentary domain that originated in partially different paleoenvironments and were later separated by tectonic contacts. Since each Tectonostratigraphic Unit can only be defined by comparison with its adjoining units (now distant bodies), it cannot be defined in an absolute, unambiguous way, and, then, not expressed by axioms. Anyway, all together the axioms defined for the Lithostratigraphic, Lithotectonic and Geologic Event concepts, respectively, give the necessary and sufficient conditions to formally (and fully) encode the knowledge that is inherent to the Tectonostratigraphic Unit concept. As a consequence, the Tectonostratigraphic Unit concept was deprecated in OntoGeonous (in a way that is consistent with CGI vocabularies and INSPIRE codelists, which actually do not report such a term).

OntoGeonous has been the product of the interaction between geologists and computer scientists, who exchanged many ideas during the encoding process. From a methodological perspective, of a paramount importance has been the initial attempt of the geologist to address the construction of the terminological knowledge. This practice has brought the geologist the awareness of the difficulty of the terminological vocabulary conception; then, later, when driven by the axiomatic encoding process, led by the computer scientist, the geologist can contribute effectively to the axiom building and address effectively the viewpoint of the task at hand, namely the geomapping process. During the ontology development, an effective tool for discussion of the axiomatic encoding ongoing was the implementation of a wiki ${ }^{21}$. For each concept definition, we created a page and the most debated issues opened discussion pages with links to the most relevant sources. Now, the wiki is released as a resource for further investigation as well as a human readable version of the knowledge (cf. [11] on the importance of wiki's for knowledge creation). It reports the motivations that have driven the encoding choices, which can be susceptible of novel updates.

The formal encoding of the geological knowledge opens new perspectives for the analysis and representation of the geological systems. These often have a very complex internal setting and a large range of physical properties, acquired in distinct geochronological steps (punctuated by geologic events), but rarely fully explicitly described $[1,5,9,16]$. In fact, once that the major concepts employed in the implementation of a geological map data base are defined, with their meaning explicitly expressed through a computational ontology, the resulting formal conceptual model of the geologic system can hold across different technical and scientific communities. Furthermore, this would allow for a semi-automatic or

$\overline{{ }^{21} \text { https://www.di.unito.it/wikigeo/. }}$ 
automatic classification of the cartographic database, where a significant number of properties (attributes) of the recorded instances could be deduced (inferred) through computational reasoning.

Acknowledgements. We, the authors acknowledge a grant (direct beneficiary Dario Mimmo) for the Lagrange Project - CRT Foundation/ISI Foundation. We also thank Rossana Damiano, Claudio Mattutino, Simone Donetti, and the Technical Staff of the Computer Science Department of the University of Turin gave us support in the setup of the wiki and the semantic server. We thank ARPA Piemonte for the collaboration on the implementation of the application interface with the semantic server (http:// webgis.arpa.piemonte.it/Geoviewer2D).

\section{References}

1. Balestro, G., Piana, F.: The representation of geological knowledge and uncertainty in databases of GIS geological maps. Boll. Soc. Geol. Ital. (Ital. J. Geosci.) 126(3), 487-495 (2007)

2. Barahmand, S., Taheyrian, M., Al-Ashri, S.: Upadhyay: a survey on SWEET ontologies and their applications. Technical report, University of Southern California (2010). http://www-scf.usc.edu/taheriya/reports/csci586-report.pdf

3. Berners-Lee, T., Hendler, J., Lassila, O.: The semantic web. Sci. Am. Mag. 284, 28-37 (2001)

4. Bizer, C., Heath, T., Berners-Lee, T.: Linked data - the story so far. Int. J. Semant. Web Inf. Syst. 5(3), 1-22 (2009)

5. Brodaric, B., Gahegan, M., Harrap, R.: The art and science of mapping: computing geological categories from field data. Comput. Geosci. 30(7), 719-740 (2004)

6. Cipolloni, C., Pantaloni, M.: La standardizzazione dei dati geologici. mem. descr. carta geol. d'it. In: La Cartografia del Servizio Geologico d'Italia, vol. 100, pp. 260-263 (2016)

7. Durbha, S., King, R., Shah, V., Younan, N.: A framework for semantic reconciliation of disparate earth observation thematic data. Comput. Geosci. 35, 761-773 (2009)

8. Fox, P., McGuinness, D.L., Cinquini, L., West, P., Garcia, J., Benedict, J.L., Middleton, D.: Ontology-supported scientific data frameworks: the virtual solarterrestrial observatory experience. Comput. Geosci. 35, 724-738 (2009)

9. Frodeman, R.: Geological reasoning: geology as an interpretive and historical science. Geol. Soc. Am. Bull. 107(8), 960-968 (1995)

10. Heath, T., Bizer, C.: Linked data: evolving the web into a global data space. Synth. Lect. Semant. Web: Theory Technol. 1, 1-136 (2011)

11. Howard, A.S., Hatton, B., Reitsma, F., Lawrie, K.I.: Developing a geoscience knowledge framework for a national geological survey organisation. Comput. Geosci. 35, 820-835 (2009)

12. Huber, R., Klump, J.: Charting taxonomic knowledge through ontologies and ranking algorithms. Comput. Geosci. 35, 862-868 (2009)

13. Laxton, J.L.: Geological map fusion: Onegeology-Europe and inspire. Geol. Soc. Lond. Spec. Publ. 408 (2016). http://sp.lyellcollection.org/content/early/2016/ 09/27/SP408.16.abstract 
14. Lombardo, V., Piana, F., Fioraso, G., Irace, A., Mimmo, D., Mosca, P., Tallone, S., Barale, L., Morelli, M., Giardino, M.: The classification scheme of the piemonte geological map and the ontogeonous initiative. Rendiconti Online Società Geologica Italiana 39, 117-120 (2016)

15. Lombardo, V., Piana, F., Mimmo, D.: Semantics-informed geological maps: conceptual modeling, knowledge encoding, workflow, and application. Comput. Geosci. (2017, resubmitted)

16. Loudon, T.V.: Geoscience after IT: part J human requirements that shape the evolving geoscience information system. Comput. Geosci. 26(3A), A87-A97 (2000)

17. Ludäscher, B., Lin, K., Brodaric, B., Baru, C.: GEON: toward a cyberinfrastructure for the geosciences-a prototype for geological map interoperability via domain ontologies. In: Soller, D. (ed.) Digital Mapping Techniques, 2003-Workshop Proceedings, pp. 223-229. Millersville, PA, USA (2003)

18. Ma, X.: Ontology spectrum for geological data interoperability. Ph.D. thesis, University of Twente (2011)

19. Ma, X., Carranza, E., Wu, C., der Meer, F.V.: Ontology-aided annotation, visualization and generalization of geological time scale information from online geological map services. Comput. Geosci. 40, 107-119 (2012)

20. Narock, T., Szabo, A., Merka, J.: Using semantics to extend the space physics data environment. Comput. Geosci. 35, 791-797 (2009)

21. Piana, F., Fioraso, G., Irace, A., Mosca, P., d'Atri, A., Barale, L., Falletti, P., Monegato, G., Morelli, M., Tallone, S., Vigna, G.B.: Geology of piemonte region (NW Italy, Alps-apennines interference zone). J. Maps 13(2) (2017). https://doi. org/10.1080/17445647.2017.1316218

22. Piana, F., Lombardo, V., Mimmo, D., Mulazzano, E., Barale, L., D’Atri, A., Irace, A., Morelli, M., Mosca, P., Tallone, S.: The geodatabase of the piemonte geological map: conceptual design for knowledge encoding. Rendiconti Online Società Geologica Italiana 42, 85-89 (2017)

23. Raskin, R., Pan, M.: Semantic web for earth and environmental terminology. Comput. Geosci. 31(9), 1119-1125 (2005). SWEET - http://ceur-ws.org/vol-83/sia_7. pdf

24. Richard, S., Allison, L.: Capitalizing on global demands for open data access and interoperability - the USGIN story. In: EGU2016-9540, Geophysical Research Abstracts, vol. 18, EGU General Assembly (2016). http://meetingorganizer. copernicus.org/EGU2016/EGU2016-9540.pdf

25. Soller, D.R., Lindquist, T., Matti, J.: Field description of the scientific and locational accuracy of geological features. In: Soller, D.R. (ed.) DMT 2002 - USGS Open-File Report, 02-370, pp. 33-40. USGS (2002)

26. Woodcock, R., Simons, B., Duclaux, G., Cox, S.: Auscope's use of standards to deliver earth resource data. In: Geophysical Research Abstracts 12, EGU General Assembly. p. 1556 (2010) 